\title{
RADIOLOGY IN THE DIAGNOSIS OF DUODENAL ULCER.
}

\author{
By H. CECIL BULL, M.A., M.B., M.R.C.P. \\ (Physician and Hon. Radiologist, Royal Waterloo Hospital. Hon. Radiologist, \\ Southend-on-Sea General Hospital.)
}

\section{Etiology.}

It is easier to suffer for sin when we have sinned, but duodenal ulcer falls alike on the just and the unjust. We know a good deal about the diagnosis, something about the treatment, nothing about the cause. It is a disease widespread among civilised peoples, very chronic and probably accompanying some victims throughout life. It tends to remit and relapse and even to heal spontaneously. Those are the simple facts. There are many theories.

The most interesting comparative work is the experimental work of Van Wagoner and Churchill (I932), Mann and Bollman (I935) who showed that peptic ulcer is produced in dogs by continued administration of cinchophen but that the ulcers heal rapidly when the drug is withdrawn. The further work of Stalker (I937) shows that ulcers do not occur if the dogs are fed with milk and alkalis at the same time as cinchophen. Neither do they occur in the presence of gastroenterostomy. Histidine has no preventive action.

Reasoning from our knowledge of chronic ulcers elsewhere in the body we should, and we do, look for an infection-especially a specific infection-or a source of chronic irritation, or interference with the blood supply. Yet all these accepted causes for chronic ulcer fall down before the peculiar individual behaviour of the peptic ulcer in remitting spontaneously. There is also a certain-if unconservative-tendency for the relapses to be seasonal. Most easily acceptable to the facts in the theory of deficiency in some essential food factor. If this should be true it is plain that we are still in the outer darkness concerning the food factors essential to health, since treatment with the known vitamins has not so far been successful.

\section{Diagnosis.}

In the diagnosis of duodenal ulcer the history and symptomatology are important in estimating the duration and activity. Occult blood in the stools shows loss of blood, and the blood count indicates how far the blood-forming elements are failing to make good. The test meal is a record of gastric acidity and the rate of gastric digestion. But radiology establishes the diagnosis.

Radiological diagnosis. The single fact upon which the diagnosis by $\mathrm{X}$-rays depends is that a peptic ulcer is a departure from the anatomical normal. An ulcer is a localised destruction of mucous membrane, gives rise to inflammatory thickening of the adjacent mucosa and submucosa and causes irregular contraction of the muscular coat. These anatomical changes are recognised radiologically as deformity in the mucous membrane and deformity in the outline of the bariumfilled duodenum. In some cases the deformity of the mucous membrane predominates and in others the outline is so deformed as to obliterate the pattern of the mucous membrane. Careful screen examination rarely fails to show these deformities, and films taken with compression to show the mucous membrane will in many cases-according to the position of the ulcer and the skill of the operator-show the ulcer crater. L. G. Cole (I9I4) of New York first recognised duodenal deformity as a constant sign of duodenal ulcer. Forssell (I923) of 
Stockholm drew attention to swelling and deformity in the pattern of the mucous membrane folds, Akerlund (I923) to the combination of niche and incisura. Berg (I93I) and Chaoul (I928) in Germany, Cordiner \& Calthrop (I936) in England have insisted on visualisation of the crater or the scar of its healing.

The pioneer work in demonstrating small ulcer craters in the duodenal cap stands in the name of Berg who postulated that the deformities of a crater in mucous membrane and outline of the duodenal cap could be shown in two planes (Fig. 2.). Since the majority of ulcers occur on the posterior or anterior walls, or both, the two views are normally the flat and the lateral. In the lateral position the projection of the ulcer crater is identified at the screen examination and immediately recorded on small films. In the flat position graduated compression is used to spread the barium emulsion and show the mucous membrane folds swollen and deformed in relation to the barium-containing crater.

The necessary equipment consists of a high-powered unit with devices for compression, rapid interchange of cassettes and change of current and voltage, and a table on which the patient can be moved from the erect to the reclining position if desired.

It is not however possible to show the crater with precision in every case. The high duodenal cap out of reach for compression, the irritable cap with pyloric spasm-difficult to fill and expelling the barium as soon as filled, the broad and rather redundant second and third parts of a duodenum which retain barium and obscure the exact view: these are some of the difficulties which alone make accurate recording by films of the screen image hard to achieve.

Åkerlund recorded a niche in 60 per cent. of duodenal ulcers. Geyman (I932) in 50 per cent. to 60 per cent. The accuracy in diagnosis of duodenal ulcer without film recording of the crater in every case is over 90 per cent. Kirklin (I93I) found that without special methods the ulcer crater was shown on films in 15 per cent. but his total diagnostic accuracy was 95 per cent. Carman (I920) reported 95 per cent. Bull \& Derry (I936) 9I per cent.

While accepting film demonstration of the crater in two planes as the ideal to be attempted in every case, my view is that the screen examination is paramount and that the outline deformity once seen can be reproduced on films. In many cases the ulcer crater can be reproduced but even with the best technique it is not always possible. Deformity of the duodenal cap (Fig. 3.) is still an index of duodenal ulcer with certain exceptions. The differential diagnosis can be made between those exceptions, and with the clinical evidence an accurate diagnosis reached.

Duodenal deformity. Duodenal deformity is a sign of duodenal ulcer with certain exceptions:- -

I. Adhesion of the duodenum to the gall-bladder.

2. Deformity from operations on the pylorus and duodenum or from adhesions following surgery in the upper abdomen.

3. Deformity does not indicate the state of activity of an ulcer. It persists in many cases when the ulcer is healed.

4. Spastic contraction which is inconstant, not fixed, associated with irritability. 


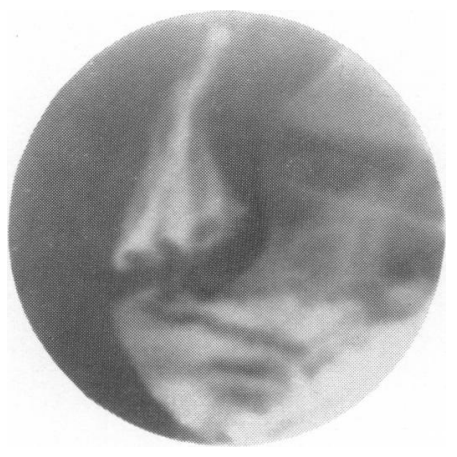

Fig. I.-Normal duodenal cap.
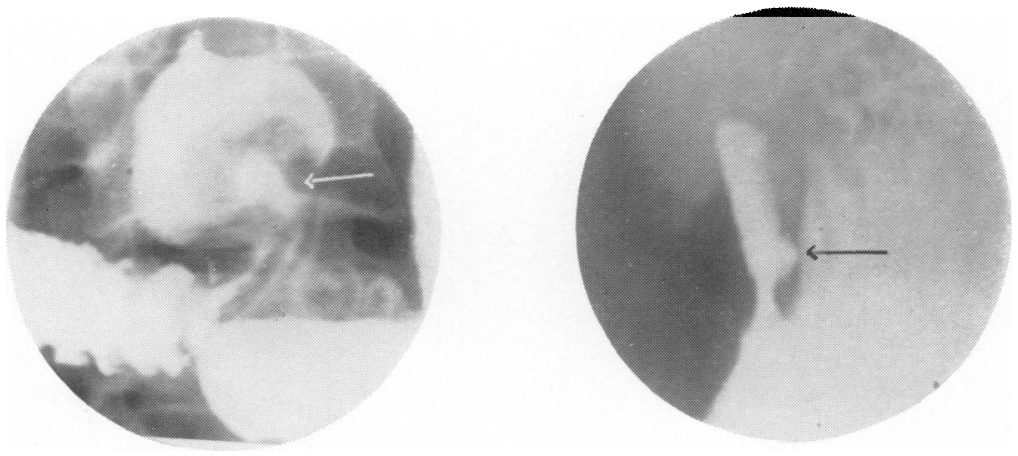

Fig. 2. A. \& B.-Anteroposterior and lateral views of a crater with little other deformity.

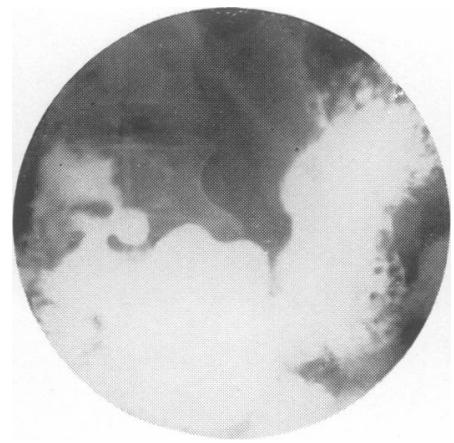

Fig. 3.-Deformity of the duodenal cap.

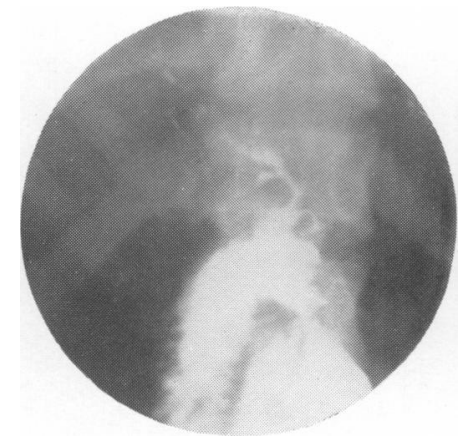

Fig. 4.-Deformity of cap from ulceration of a gall stone. Barium tracking into the gallbladder and bile ducts.

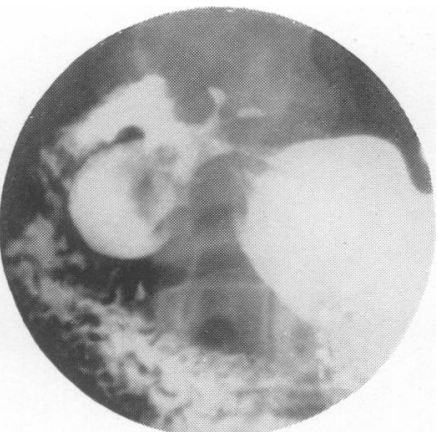

Fig. 5.-Gross deformity of the duodenal cap. 
(I). Adhesions to the gall-bladder. These are inflammatory adhesions. Occasionally a gall-stone will ulcerate through and be discharged into the duodenum (Fig. 4.). The deformity of adhesions is often a flattening of the outer border associated with irritability of the duodenal cap. Local tenderness is the rule. The symptoms suggest gall-bladder disease and the diagnosis is confirmed by cholecystography.

(2). Postoperative deformities. These deformities vary according to the nature of the operation and site of the adhesions but in general they are distortions of the cap rather than spastic contraction of the circular muscle. The history of operation should prevent a mistaken diagnosis.

(3). Activity of duodenal ulcer. This question justifies the claim that the ulcer crater should be demonstrated. I formerly held the view that demonstration of the crater was too inaccurate, and interpretation of the shadows of mucous folds too liable to error and the enthusiasm of the observer. But I now think that this view was wrong. Beyond doubt the liability to error exists but the principle of striving for the ideal is right and even though the percentage of success in showing a crater is low-and it varies in different hands-the attempt should be made in every case. I do not, however, hold the view that duodenal ulcer can be diagnosed only when the crater is demonstrated on films.

Positive identification of the ulcer crater-and it is frequently on both the anterior and posterior walls of the duodenum-is helpful in following the progress of an ulcer under treatment. Once identified beyond question the patient can be re-examined at intervals to show the contraction in size of the ulcer crater. Reexamination is also called for when there has been any doubt in the mind of the radiologist. Cases vary, there are always difficult ones and if they should come at a time when judgment is not at its keenest, judgment may err. A period of treatment between the examinations changes the picture as regards the extrinsic factors. Much of the irritability associated with a raised acidity has disappeared, the patient is accustomed to the examination, the radiologist is forewarned of his difficulties and can look ahead to meet them. The chances of error in a second examination are very small.

(4). Inconstant spastic contractions of an irritable duodenum. In this, demonstration of the ulcer crater alone can make the positive diagnosis of duodenal ulcer. Such irregular contractions may be due to adhesions as in (3), but they may also be a sign of duodenitis. Pathologically, duodenitis shows an increased vascularity and an odema of the mucous membrane, sometimes with a localised erosion. It is associated with increased gastric acidity and is usually reflex from irritation lower in the alimentary canal-inflammation of the ileum, appendix or colon. These cases show duodenal irritation. Failure to see a crater does not exclude ulcer but the treatment is different. The indication is to find and remove the cause. The duodenitis will then settle down under simple ulcer treatment.

Evidence of healing of duodenal ulcer. It is encouraging for the physician to see a positive crater disappearing gradually at re-examinations during the course of treatment. It shows good treatment. It does not indicate cure. A bout of injudicious eating and drinking can restore the crater to its previous size in a few days. Duodenal ulcers do not heal easily, they start well but tend to recur after a lapse of time even among the most careful patients. We can speak of them as inactive but having regard to their peculiar qualities it is optimistic and rather 
unwise to use the word cure. Perhaps the shortest time is two years. Two Springs and Autumns without a relapse. Even so they may recur after a remission of years.

In radiology the ulcer which shows a defined crater and little other deformity. is the easiest to watch in the process of healing. But it is also the least common. Total deformity of the cap is commoner than the niche and incisura, and even after prolonged treatment and clinical "cure" the deformity persists (Fig. 5.). Theoretically the scarring of a healed ulcer should be identified in the mucous membrane but in practice this is rarely achieved. It is possible with a duodenal ulcer with well defined crater and little other deformity, but in most cases-where total deformity is present - the deformity obscures the detail of the mucous membrane picture. No one has yet satisfactorily explained duodenal deformity. Due in part to spasm it should in part relax when the inflammation is arrested and in process of healing. Surgery has shown that the radiological deformity is often out of proportion to the size of the ulcer and extent of organic involvement of the walls. Conversely too radiology shows that a large crater may be found in a duodenal cap not otherwise deformed whereas it is difficult to identify the crater in the presence of gross deformity.

It is as uncertain for the radiologist as for the clinician to determine when an ulcer is cured. A diminished irritability and some slight lessening of the spastic deformity may be seen at re-examination after a period of treatment but I doubt whether a really deformed cap (Fig. 5.) ever regains its normal shape with no other indication than the scar of its healing. There is something here that we do not understand, an uncommon instance of pathology, clinical medicine and radiology not being quite in step together. Clearly we have not reached the truth. The explanation which fits the case is that these duodenal ulcers with gross deformity of the cap do not heal, merely undergo long periods of remission: After studying and following up duodenal ulcers for a number of years I believe this to be true. They are enormously improved by a strict course of treatment, and provided the patient maintains care in after treatment life is tolerable and the symptoms no more than a mild dyspepsia. Occasional relapses are not usually severe and are controlled by a short course of treatment and more discipline in the regime. But the radiological deformity persists and with it I believe the smouldering activity of an ulcer quiet but unhealed. The view is not a new one, it was the basis of the surgical approach to duodenal ulcer expressed epigrammatically by J. B. Deaver (I922) "once an ulcer always an ulcer." I do not feel the same conviction about the ulcers with defined craters and little other deformity, they are not so common and I have not had the same experience in following them over a long period or the interested outlook which I have now. It is my impression that this may be the ulcer which heals completely. (Fig 2.).

\section{Conclusion.}

The radiological findings - whether they be deformity of outline or mucous membrane - with or without visible crater-must be assessed in final judgment by the clinician to whom these findings are a link-perhaps the most valuable linkin the chain of evidence.

An ulcer is an anatomical deformity which can be recognised by radiology in a high percentage of cases, the exact cause of the deformity can be determined by seeing the crater in a further percentage, and the final picture of diagnosis, prognosis and the treatment suited for the particular case is painted in with very 
great accuracy by the clinician with his knowledge of the history, physical examination and the other laboratory tests. When all are assessed together the errors in diagnosis of duodenal ulcer should be negligible.

It was said at the beginning that duodenal ulcer is a very chronic disease often persisting throughout life with peaks of relapse, and with periods of remission-after treatment, but sometimes spontaneously. It is during the peaks of relapse that most cases are seen by radiologists. But failure to demonstrate an ulcer crater in a deformed duodenal cap will not mislead the clinician, who has ample evidence of activity. Neither will the demonstration of scarring without crater alone convince him that the ulcer is cured. The radiologist supplies the illustrations but the clinician tells the story. 